\title{
USO DO SISTEMA DE INFORMAÇÃO GEOGRÁFICA E DADOS DE SENSORIAMENTO REMOTO NO PROCESSO DE MODELAGEM ESPACIAL MULTICRITÉRIO PARA A DEFINIÇÃO DE DIRETRIZES DE LINHAS DE TRANSMISSÃO DE ENERGIA ELÉTRICA
}

\author{
USE OF THE GEOGRAPHIC AND DATA INFORMATION SYSTEM OF REMOTE SENSING TO \\ THE PROCESS OF MULTICRITERIA SPATIAL MODELING FOR THE DEFINITION OF \\ GUIDELINES OF ELECTRIC POWER TRANSMISSION LINES
} \author{
Paraná. E-mail fabianofreiman@ufpr.br; danielsantos@ufpr.br

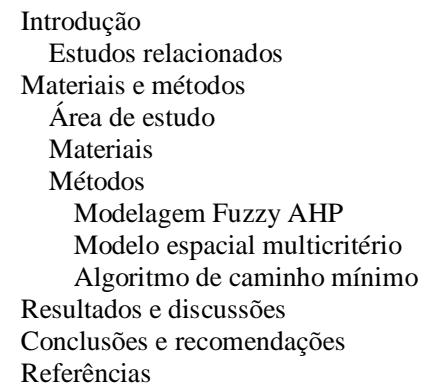

Fabiano Peixoto FREIMAN, Daniel Rodrigues dos SANTOS

Programa de Pós-Graduação em Ciências Geodésicas. Universidade Federal do Paraná. Rua XV de Novembro, 1299, Curitiba -

RESUMO - No Brasil. devido ao crescimento econômico e populacional a construção de novas linhas de transmissão (LT) de energia elétrica é essencial. Este processo é complexo visto que é necessário o equacionamento de fatores conflitantes (fatores ambientais, sociais e econômicos) para a definição do traçado de uma diretriz que minimize os impactos gerados pelo empreendimento. Neste contexto, este artigo apresenta uma metodologia para a definição do traçado de uma diretriz de LT de energia elétrica baseado na utilização de ferramentas do Sistema de Informação Geográfica (SIG), dados de Sensoriamento Remoto, a lógica Fuzzy e o Método de Análise Multicritério (MAM) Analytical Hierarchy Process (AHP). Os resultados demonstraram que a técnica emprega é eficiente e adequada para a determinação automática de diretrizes de LT, facilitando o processo de planejamento de um novo empreendimento, minimizando erros de planejamento e possíveis retrabalhos.

Palavras-chaves: Planejamento. AHP. Fuzzy. SIG. Linhas de transmissão.

\begin{abstract}
In Brazil, due to economic and population growth, the construction of new electric power transmission lines (LT) is essential. This process is complex since it is necessary to equate conflicting factors (environmental, social and economic factors) in order to define a guideline that minimizes the impacts generated by the project. In this context, this article presents a methodology for the definition of the layout of an electric power LT guideline based on the use of tools from the Geographic Information System (GIS), Remote Sensing data, fuzzy logic and the Multicriteria Analysis Method (MAM) Analytical Hierarchy Process (AHP). The results showed that the technique employed is efficient and adequate for the automatic determination of LT guidelines, facilitating the planning process of a new venture, minimizing planning errors and possible rework.
\end{abstract}

Keywords: Planning AHP. Fuzzy. SIG. Transmission lines.

\section{INTRODUÇÃO}

O planejamento de diretrizes de linhas de transmissão (LT) de energia elétrica é definido como o estágio antecessor ao projeto de uma nova LT, no qual o planejador decide a geometria do traçado e as áreas que serão atravessadas pelo empreendimento (Monteiro et al., 2005). O processo de definição de uma diretriz é complexo devido à dimensão linear da LT, que geralmente atravessa diferentes munícipios e/ou estados com interesses políticos divergentes. Ademais, áreas de preservação ambiental, remanescentes florestais e outros tipos de áreas protegidas por lei devem ser evitadas, além da consideração de barreiras físicas como o percentual de declividade, rios, áreas alagadas, etc (Bachmann et al., 2018). Em abordagens convencionais, o planejamento de diretrizes de LT é realizado de forma manual, fato que pode resultar em inconsistências. Segundo a Empresa de Pesquisa em Energia Elétrica (EPE, 2005), é indicado utilizar um enfoque estratégico considerando fatores sociais, ambientais e econômicos. Neste contexto, a aplicação de Métodos de Análise Multicritério (MAM) associados ao Sistema de Informação Geográfica (SIG) auxiliam na compreensão da relação espacial entre os fatores (Bagli et al., 2015) e obtenção de resultados fundamentados em modelos espaciais realistas (Malczewski, 2006). Vale ressaltar que 
este trabalho apresenta parte dos resultados alcançados durante a execução do projeto de $\mathrm{P} \& \mathrm{D}$ PD-06491-0353-2014 executado pela Universidade Federal do Paraná - UFPR para a COPEL Geração e Transmissão S.A. no âmbito do Programa de Pesquisa e Desenvolvimento Tecnológico do Setor de Energia Elétrica regulamentado pela Agência Nacional de Energia Elétrica ANEEL.

\section{Estudos relacionados}

O processo de definiç̧ão do traçado de diretrizes de LT é clássico em projetos de LT e frequentemente são apresentados métodos que visam a automatização desta etapa e minimização de inconsistências. Shu et al. (2012) propuseram um método para a definição de diretrizes de LT considerando variáveis ambientais baseada em programação linear associada ao SIG. Monteiro et al. (2005) utilizaram dados geográficos associados a programação dinâmica (PD).

Lima et al. (2016) utilizaram o algoritmo de Dijkstra (1959) para a definição de diretrizes de LT. A contribuição do trabalho foi a realização das ponderações baseadas nos custos monetários de construção, buscando a minimização de inconsistências. Thiam \& Demarco (2016) utilizaram o método Analytic Hierarchy Process (AHP) associado a técnica Delphi para validar a consistência dos pesos atribuídos aos critérios sociais, ambientais e econômicos.

Bachmann et al. (2018) desenvolveram uma ferramenta de análise espacial para a definição do traçado automático de diretrizes de LT. Foram utilizados dados relacionados a eficiência econômica, uso e ocupação do solo e áreas de preservação ambiental. Bagli et al. (2011) realizaram o processo de definição automática de diretrizes de LT a partir da análise de fatores ambientais, sociais e econômicos. O traçado otimizado, em ambos os trabalhos, foi definido a partir da aplicação do algoritmo de Dijkstra (1959).

Dentre os trabalhos citados, observa-se que a utilização de MAM para a realização do processo de definição de pesos é frequentemente empregada. Veronesi et al. (2017) mencionam que o principal problema relacionado aos MAM é a subjetividade, uma vez que a ponderação dos fatores geográficos é definida a partir das experiências de especialistas.

Segundo Papaioannou et al. (2014), a associação de MAM (construção de um modelo hibrido) possibilita a minimização da subjetividade do processo e minimiza as inconsistências da Modelagem Espacial Multicritério (MEM).

Neste contexto, este trabalho propõe a realização da MEM para a definição de diretrizes de LT de energia elétrica utilizando uma abordagem hibrida (teoria Fuzzy e o método Analytic Hierarchy Process (AHP), desenvolvido por Saaty (1980)). A partir deste trabalho, objetiva-se construir um modelo de adequação ao recebimento de uma nova LT.

A contribuição do estudo está relacionada a aplicação de um método que pode ser adaptado e replicado em outras regiões, possibilitando a geração de diretrizes de faixas de domínio em projetos de forma rápida e precisa dentro dos parâmetros estipulados, minimização de tempo para a sistematização de uma diretriz, tanto para a participação das concessionárias em leilões promovidas pela Agencia Nacional de Energia Elétrica (ANNEL), quanto para a definição da diretriz do traçado do projeto.

Além disto, neste trabalho foram empregados dados geográficos obtidos gratuitamente, e utilizados softwares de análise espacial gratuitos, o que não acarreta na adição de custos no processo de planejamento e viabiliza a replicação da metodologia proposta dentro de uma área de interesse.

\section{MATERIAIS E MÉTODOS}

Nesta Seção são descritos: a área de estudo para aplicação da metodologia proposta, os materiais utilizados para a realização do processo de otimização do traçado de LT de energia elétrica e a estrutura metodológica.

\section{Área de estudo}

Para avaliar o método proposto foi realizado um estudo de caso, sendo escolhida uma área que apresentasse uma LT de energia elétrica existente, possibilitando a validação dos resultados. A região de estudo está localizada no estado do
Paraná e compreende a área de abrangência da LT de energia elétrica $(525 \mathrm{kv})$ que liga as Subestações de Foz do Iguaçu a Subestação de Guaíra (Figura 1).

\section{Materiais}

Para a realização do processo de otimização do traçado de uma diretriz de LT, realizou-se uma pesquisa nos trabalhos relacionados ao tema e na legislação brasileira para selecionar os critérios que influenciam no processo de definição do traçado locacional de uma LT de energia elétrica. 


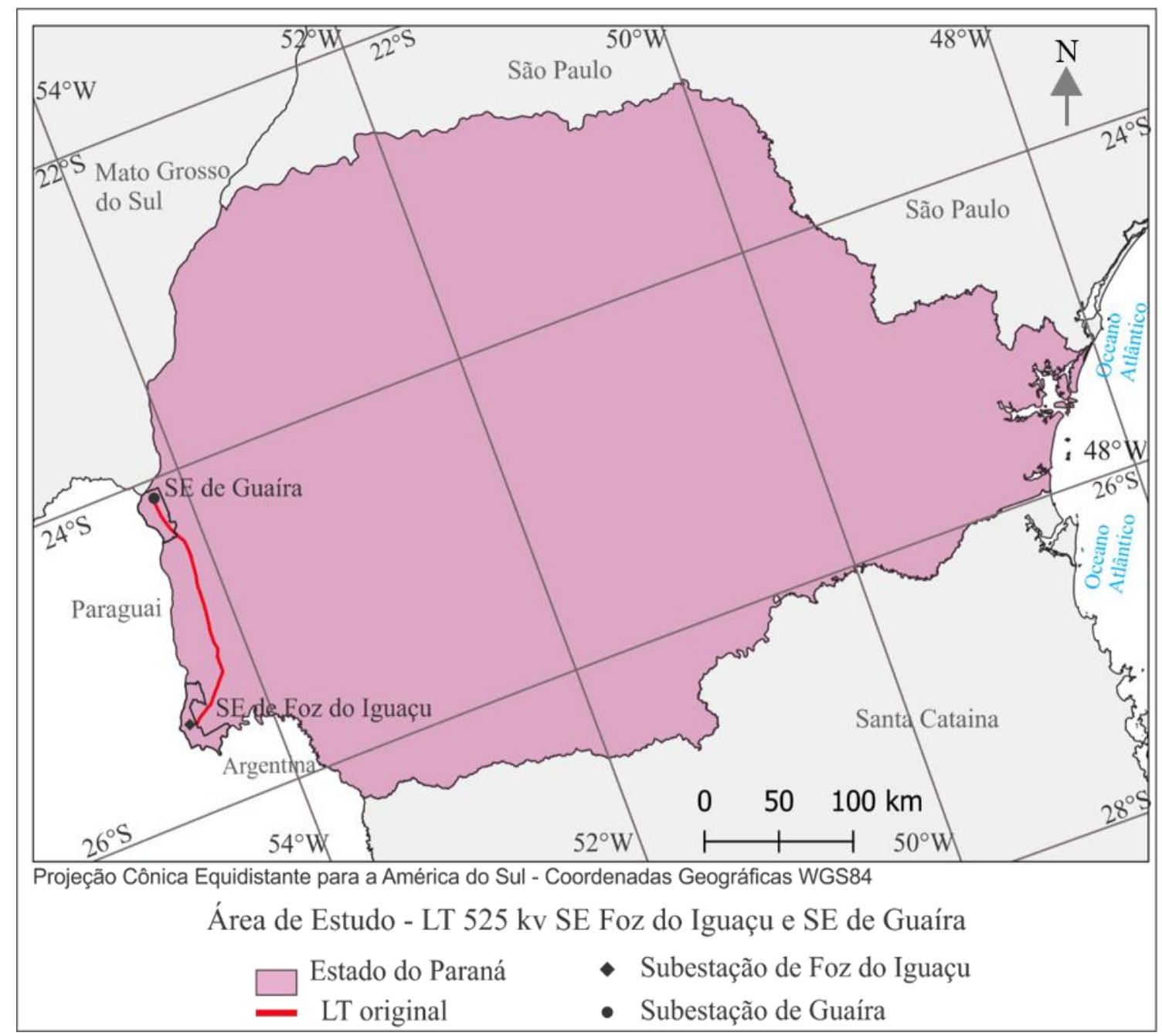

Figura 1. Área de estudo no estado do Paraná, com localização da LT considerada.

Os dados utilizados neste trabalho (critérios) são apresentados no Quadro 1 e foram adquiridos por meio de órgãos públicos disponibilizados gratuitamente em plataformas online.
Os critérios utilizados foram divididos em três grupos: ambiental, social e econômico, conforme o processo de planejamento estratégico para a definição do traçado de uma nova LT definido por EPE (2005).

Quadro 1. Critérios considerados

\begin{tabular}{|l|l|l|l|l|}
\hline \multicolumn{1}{|c|}{ Grupo } & \multicolumn{1}{|c|}{ Fator } & \multicolumn{1}{c|}{ Escala } & \multicolumn{1}{c|}{ Formato } & \multicolumn{1}{c|}{ Fonte } \\
\hline \multirow{4}{*}{ Ambiental } & Unidade de conservação & Não Informada & Vetorial & IAP (2018) \\
\cline { 2 - 5 } & Remanescentes florestais & $1: 40000$ & Vetorial & IAP (2018) \\
\cline { 2 - 5 } & Terras Indigenas & $1: 40000$ & Vetorial & INCRA (2018) \\
\hline \multirow{5}{*}{ Social } & Uso e ocupação do solo & Não informado & Matricial & - \\
\cline { 2 - 5 } & Áreas Urbanas e periurbanas & $1: 60000$ & Vetorial & GEOINFO (2018) \\
\cline { 2 - 5 } & Assentamentos Rurais & Não Informado & Vetorial & INCRA (2018) \\
\hline \multirow{5}{*}{ Econômico } & Declividade & $1: 60000$ & Matricial & Miranda (2005) \\
\cline { 2 - 5 } & $\begin{array}{l}\text { Paralelismo com outros } \\
\text { empreendimentos lineares }\end{array}$ & Não informado & Vetorial & $\begin{array}{l}\text { EPE (2018) E DNIT } \\
(2018)\end{array}$ \\
\cline { 2 - 5 } & Áreas alagadas & $1: 50000$ & Vetorial & IAP (2019) \\
\hline
\end{tabular}

Os critérios ambientais foram definidos para a preservando a fauna e a flora da região. Segundo realização de um projeto ambientalmente a Portaria $\mathrm{n}^{\circ} 421$, de 26 de outubro de 2011, áreas sustentável, visando a inclusão de políticas de de preservação ambiental, quilombolas e mitigação de interferência ao meio ambiente, indígenas devem ser evitadas. Neste trabalho 
foram considerados 3 critérios ambientais: remanescentes florestais, áreas de preservação ambiental e áreas indígenas.

Os critérios sociais dizem respeito a como a nova LT pode afetar a população residente na área de influência do empreendimento. Segundo Grassi et al. (2014) a construção de uma nova LT causa poluição visual, desvalorização de imóveis, riscos à saúde da população mais próxima ao empreendimento, além da necessidade de desapropriações de áreas agrícolas e residenciais, causando custos adicionais a concessionária. Neste trabalho foram considerados 3 fatores sociais: uso e ocupação do solo, áreas urbanas e periurbanas e assentamentos rurais. Destaca-se que para a construção das áreas periurbanas, realizou-se a construção de Buffers ao redor das zonas urbanas, sendo consideradas 4 classes: $90 \mathrm{~m}$, $180 \mathrm{~m}, 270 \mathrm{~m}$ e $360 \mathrm{~m}$.

Por fim foram considerados critérios econômicos, os quais estão relacionados diretamente com os custos de construção de uma LT. Nesta perspectiva foram consideradas a declividade do terreno, uma vez que locais com declividades acentuadas necessitam de maior investimento para a construção de uma LT. Esta classe foi construída com base nos dados do projeto Shuttler Radar Topographic Mission (SRTM) pertencente a National Aeronautics and Space Administration (NASA), obtido no site da Empresa Brasileira de Pesquisa e Agropecuária (EMBRAPA) (Miranda, 2005).

Além disso, segundo a Portaria n 421 de 26 de outubro de 2011, a construção de uma LT ao longo de faixa de domínio de rodovias, ferrovias, LT já instaladas, ainda que em território quilombola, indígena ou em unidade de conservação ambiental é considerado de pequeno potencial poluidor. Neste aspecto as áreas paralelas a empreendimentos lineares (rodovias e LT) foram consideradas como um critério atrativo no processo de otimização do traçado da diretriz de uma LT. Áreas alagadas também foram consideradas, uma vez que estas apresentam custo elevado para a construção das fundações das torres que compõem o empreendimento.

Os dados adquiridos em formato vetorial foram convertidos para o formato matricial, para possibilitar a realização do processo de álgebra de mapas. Devido as escalas de aquisição dos dados não serem coincidentes, necessitou-se compatibilizá-las para uma escala menos detalhada. (1:100.000). Os dados foram referenciados ao Sistema de Referência Geocêntrico para as Américas na época 2000 (SIRGAS 2000), sendo utilizada a Projeção Cônica Equidistante para a América do Sul (EPSG: 102032). Os processamentos foram realizados nos softwares gratuitos QGIS 3.3.6 e o Geographic Resources Analysis Support System (GRASS 7.6.1).

\section{Métodos}

Nesta Seção é apresentada a metodologia utilizada para a realização do processo de definição automática do traçado de uma diretriz de LT. A metodologia foi dividida em 4 etapas, conforme ilustrado na Figura 2.

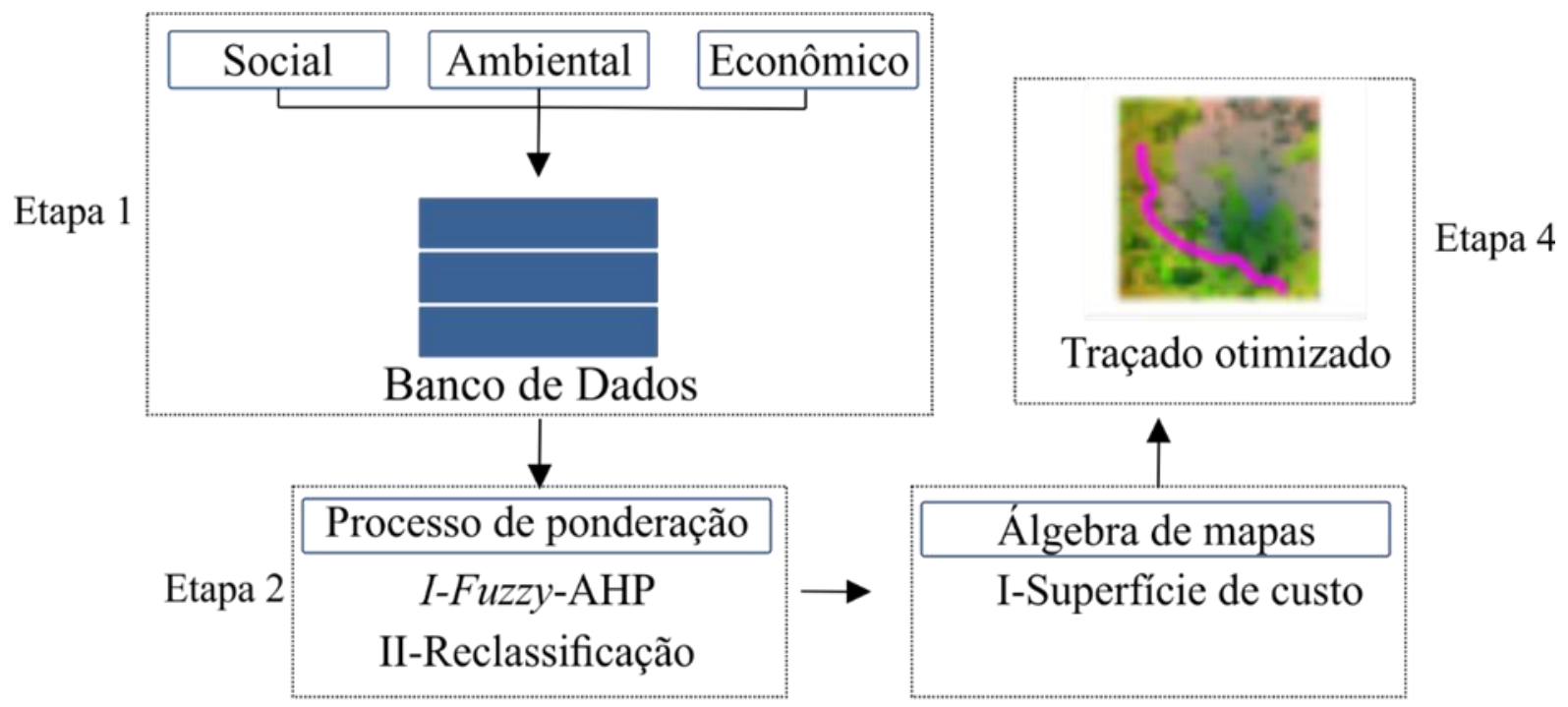

Etapa 3

Figura 2. Fluxograma metodológico. 
A Etapa 1 da metodologia proposta consiste no pré-processamento dos dados geográficos. Nesta etapa foi realizado a compatibilização do sistema de referência e projeção cartográfica, além de processamentos preliminares como a rasterização dos dados vetoriais e compatibilização das escalas das camadas utilizas para a construção do MEM. Com o banco de dados geográfico construído, foi iniciado o processo de ponderação dos critérios e subcritérios.

Os softwares QGIS 3.3.6 e o GRASS 7.6.1 foram aplicados para a realização da reclassificação dos dados de acordo com os pesos obtidos na Etapa 2, bem como a construção da superfície de custo a partir da soma ponderada das camadas matriciais (processo de álgebra de mapas (Etapa 3)). Por fim, na Etapa 4, foi aplicado o algoritmo de Dijkstra (1959) para a determinação do traçado otimizado.

\section{Modelagem Fuzzy AHP}

Nesta abordagem os dados geográficos foram estruturados em um problema de decisão hierárquico, sendo realizada a análise da influência de cada critério selecionado para a realização do processo de modelagem espacial multicritério.

No processo de ponderação é necessário a realização de comparações pareadas segundo a concepção de especialistas acerca da problemática a ser modelada, gerando uma matriz quadrada e reciproca, denominada de matriz de comparação (A).

Para a realização do processo de ponderação, utilizou-se a escala Fuzzy de importância relativa, como observa-se na Tabela 1 .

Tabela 1. Escala Fuzzy

\begin{tabular}{l|l}
\hline Importância & Escala \\
\hline Igual & $(1,1,1)$ \\
\hline Moderadamente maior & $(2,3,4)$ \\
\hline Fortemente maior & $(4,5,6)$ \\
\hline Muito fortemente maior & $(6,7,8)$ \\
\hline Extremamente maior & $(9,9,9)$ \\
\hline Valores intermédiarios & $(1,2,3)$ \\
& $(3,4,5)$ \\
& $(5,6,7)$ \\
& $(7,8,9)$ \\
\hline
\end{tabular}

A partir do julgamento pareado de 3 especialistas, obteve-se os valores de prioridade entre os critérios, identificando aqueles com maior influência no processo de definição do traçado de diretrizes de LT. Para representar os níveis de importância foram aplicados números Fuzzy triangulares $((M))$, contabilizando-se os julgamentos de especialistas e aplicando operações aritméticas simples para definir os pontos mínimo $(l)$, intermediário $(m)$ e máximo (u). Um número Fuzzy triangular $(M(l, m, u))$ é dado pela Equação 1:

$$
\mu(x)=\left\{\begin{array}{c}
\frac{x-1}{m-1} \text { se } x \in[l, m] \\
\frac{x-u}{m-u} \text { se } x \in[m, u] \\
0 c c
\end{array}\right.
$$

Para a utilização dos números triangulares no método AHP, devem ser realizadas as seguintes operações:

$$
\begin{aligned}
& M_{1}\left(l_{1}, m_{1}, u_{1}\right) \oplus M_{2}\left(l_{2}, m_{2}, u_{2}\right) \\
& \lambda\left(l_{1}, m_{1}, u_{1}\right)=\left(\lambda l_{1}, \lambda m_{1}, \lambda u_{1}\right) \lambda>0 \\
& \left(l_{1}, m_{1}, u_{1}\right)^{-1}=\left(\frac{1}{u_{1}}, \frac{1}{m_{1}}, \frac{1}{l_{1}}\right)
\end{aligned}
$$

Os números Fuzzy triangulares são alocados na matriz A (Equação 5), como segue:

$$
A=\left[\begin{array}{ccc}
(1,1,1) & \ldots & \left(a_{1 j}^{1}, a_{1 j}^{m}, a_{1 j}^{r}\right) \\
\left(a_{12}^{1}, a_{12}^{m}, a_{12}^{r}\right)^{-1} & (1,1,1) & \left(a_{2 j}^{1}, a_{2 j}^{m}, a_{2 j}^{r}\right) \\
\vdots & \ddots & \vdots \\
\left(a_{1 i}^{1}, a_{1 i}^{m}, a_{1 i}^{r}\right)^{-1} & \ldots & (1,1,1)
\end{array}\right]
$$

Para obtenção dos pesos finais dos critérios Fuzzy AHP foi utilizado o método de média geométrica, conforme Equação 6:

$$
\tilde{a}_{i}=\left(\prod_{j=i}^{3} \tilde{a}_{i j}\right)^{1 / 3}
$$

$\mathrm{O}$ vetor W (Equação 7) representa o resultado do cálculo dos pesos, ou seja, contém o peso de cada critério, finalizando a etapa de ponderação Fuzzy AHP.

$$
\begin{gathered}
W_{i}=\frac{\tilde{\mathrm{a}}_{i}}{\sum_{i=1}^{3} \tilde{\mathrm{a}}_{i}} \\
W=\left\{w_{1}, w_{2}, \ldots, w_{n}\right\}
\end{gathered}
$$

Destaca-se que neste trabalho foi realizada apenas a ponderação dos critérios com o método Fuzzy AHP. Os subcritérios foram ponderados em uma escala de 1 a 10 , em que 1 significa alta adequabilidade e 10 significa baixa adequabilidade para a instalação de uma nova LT. Na próxima Seção é apresentado o processo de álgebra de mapas para a construção do modelo espacial multicritério. 


\section{Modelo Espacial Multicritério}

Esta etapa consiste na sobreposição de todos os fatores condicionantes utilizados para a construção do MEM em uma única superfície matricial, denominada de Superfície de Custo Discreto. O objetivo é unir as informações da célula de cada camada matricial em uma única, usando o processo de álgebra de mapas, como ilustrado na Figura 3.
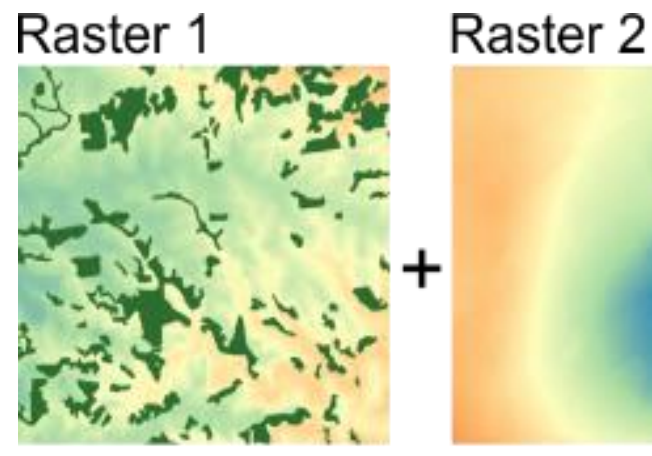
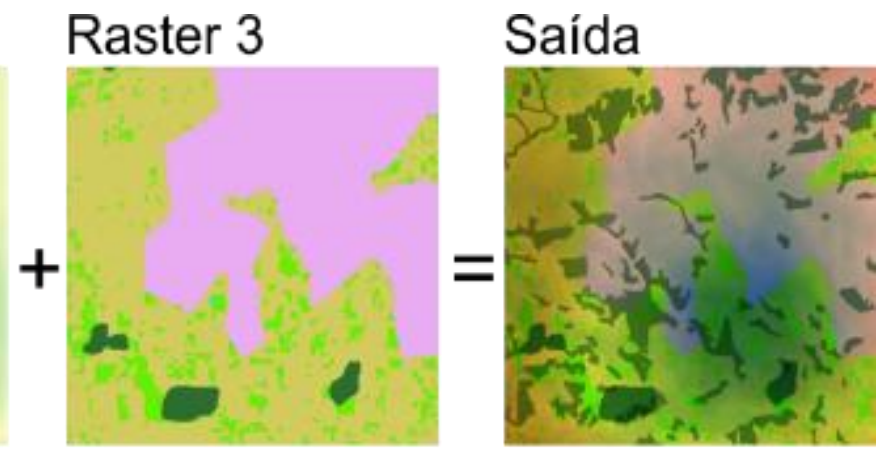

Figura 3. Processo de álgebra de mapas.

Para a construção desta superfície foi utilizada a combinação linear ponderada, como apresentado na Equação 8, como segue:

$$
S=\sum_{i}^{n} W_{i} x_{i}
$$

Em que $W_{i}$ é o peso de um fator $i, x_{i}$ o valor normalizado para o mesmo fator e n o número de fatores utilizados.

A superfície resultante foi definida em uma grade matricial $(N x M)$ com resolução espacial de $100 \mathrm{~m}$. A partir desta superfície, realizou-se o cálculo do custo acumulado entre o ponto inicial e final do traçado da diretriz da LT.

Segundo Bahmann et al. (2018), dado um ponto $s$ (início) e um ponto $d$ (destino) a superfície de adequabilidade é discretizada em um grafo ponderado e não direcionado $G=$ $(V, L)$, modelando todos os caminhos possíveis entre os pontos $s \in V$ e $d \in V$.

O conjunto de vértices $(V)$ de $G$ consiste nos centros de cada célula da superfície de adequabilidade, em que $v_{i, j} \in V \subseteq \mathbb{R}^{2}, i \in$ $\{1, \ldots, N\}, j \in\{1, \ldots, M\}$. Cada vértice de $G$ se relaciona com seus oito vértices vizinhos a partir de elementos denominados de arestas ( $L$ ) que consistem no conjunto $a_{i, j, k, l}=$ $\left\{v_{i, j}, v_{k, l}\right\}\left|v_{i, j}, v_{k, l} \in V,\right| K-1|=1 \vee| l-j \mid=$ $1\}$. Aos elementos deste conjunto são atribuídas as ponderações (positivas) médias de dois pixels vizinhos da superfície de custo discreto multiplicada pela resolução do MEM.

O caminho em $G$, entre os pontos de início e fim do traçado, segundo Bachmann et al. (2018), é uma sequência de arestas, tal que: $\mid a_{i} \cap$ $a_{i+1} \mid=1$, em que $1 \leq i \leq k-1$.

No grafo originado da Superfície de custo discreto existe um conjunto de caminhos possíveis $(\beta)$ entre os pontos $(\beta)$ entre os pontos $s=v_{i, j}$ e $d=v_{k, l} \operatorname{com} i, k \in\{1, \ldots, N\}$ e $j, l \in$ $\{1, \ldots, M\}$. O caminho ideal é aquele que apresentar o menor custo de atravessamento, definido conforme descrito na próxima Seção.

\section{Algoritmo de Caminho Mínimo}

Para a definição do traçado otimizado da diretriz da LT de energia elétrica foi aplicado o algoritmo de Dijkstra (1959). Segundo Taha (2008), este algoritmo se baseia no processo de rotulação dos vértices de um grafo ponderado (não admitindo pesos negativos), sendo executado em 3 etapas, como segue:

1) Rotula o vértice de origem $(s)$ como rotulo permanente $[0,-]$. Determina $i=1$;

2) Calcula os rótulos temporários $\left[c_{i}+c_{i j}, i\right]$ para cada vértice $j$ imediatamente adjacente ao vértice $i$ e rotulado como temporário. Caso o vértice $j$ estiver rotulado com $\left[c_{j}, k\right]$, passando por um outro vértice $k$ e se $c_{i}+$ $c_{i j}<c_{j}$, substitui $\left[c_{j}, k\right]$ por $\left[c_{i}+c_{i j}, i\right]$, os empates são resolvidos arbitrariamente;

3) $\mathrm{O}$ algoritmo encerra o processo iterativo quando todos os vértices forem rotulados como permanentes. Caso contrário, o algoritmo seleciona $\left[c_{r}, s\right]$, cujo custo de atravessamento $\left(c_{r}\right)$ é o mínimo entre todos os vértices rotulados como temporários. Determina $i=r$ e repete a etapa $i$.

\section{RESULTADOS E DISCUSSÕES}

Neste trabalho foram selecionados 9 critérios para a construção de um modelo espacial para a definição do traçado otimizado de uma LT de energia elétrica em uma área de estudo localizada 
no estado do Paraná, incluindo parâmetros sociais, ambientais e econômicos. Todos os dados foram integrados com o auxílio de ferramentas SIG.

A influência de cada critério na adequação de uma região ao recebimento de uma nova LT foi definida a partir da realização de comparações pareadas entre as 9 classes definidas, obtidas a partir da consulta a 3 especialistas do processo de definição de diretrizes de LT. Com base nestas opiniões, realizou-se a definição das ponderações de cada camada a partir da implementação do método Fuzzy-AHP em uma planilha eletrônica. Para cada grupo considerado neste trabalho foi construída uma matriz de comparação A, conforme apresentado nas Tabela 2, Tabela 3 e Tabela 4.

Tabela 2. Matriz A (Grupo ambiental): C1: Unidades de conservação, C2: Remanescentes florestais e C3: Terras indígenas.

\begin{tabular}{l|l|l|l}
\hline I & $\mathrm{C} 1$ & $\mathrm{C} 2$ & $\mathrm{C} 3$ \\
\hline $\mathrm{C} 1$ & $(1,1,1)$ & $(1,2,3)$ & $(1,1,1))$ \\
\hline $\mathrm{C} 2$ & $(0.3,0.5,1)$ & $(1,1,1)$ & $(0.3,0.5,1)$ \\
\hline $\mathrm{C} 3$ & $(1,1,1)$ & $(1,2,3)$ & $(1,1,1)$ \\
\hline
\end{tabular}

Tabela 3. Matriz A (Grupo social): C4 : Uso e ocupação do solo, C5: Áreas urbanas e periurbanas e C6: Assentamentos Rurais

\begin{tabular}{l|l|l|l}
\hline I & C4 & C5 & C6 \\
\hline C4 & $(1,1,1)$ & $(1,2,3)$ & $(0.3,0.5,1)$ \\
\hline C5 & $(0.3,0.5,1)$ & $(1,1,1)$ & $(0.3,0.5,1)$ \\
\hline C6 & $(1,2,3)$ & $(1,2,3)$ & $(1,1,1)$ \\
\hline
\end{tabular}

Tabela 4. Matriz A (Grupo econômico) C7: Declividade, C8: Empreendimentos lineares, C9: Áreas alagadas

\begin{tabular}{l|l|l|l}
\hline I & $\mathrm{C} 7$ & $\mathrm{C} 8$ & $\mathrm{C} 9$ \\
\hline $\mathrm{C} 7$ & $(1,1,1)$ & $(2,3,4)$ & $(0.3,0.5,1)$ \\
\hline $\mathrm{C} 8$ & $(0.2,0.3,0.5)$ & $(1,1,1)$ & $(0.2,0.3,0.5)$ \\
\hline C9 & $(1,2,3)$ & $(2,3,4)$ & $(1,1,1)$ \\
\hline
\end{tabular}

Observa-se que para cada processo de comparação pareada foi realizada a determinação de um número triangular Fuzzy de acordo a escala de importância apresentada na Tabela 1. A partir das matrizes de comparação pareada foi computado a média geométrica para o número Fuzzy triangular, bem como as ponderações relativas para cada critério utilizado.

A partir da matriz de comparação, para cada grupo considerado neste trabalho foi computada a média geométrica para o número Fuzzy triangular, bem como as ponderações relativas para cada critério selecionado. $\mathrm{O}$ mesmo processo foi aplicado para a definição dos pesos relacionados a cada grupo (ambiental, social e econômico) utilizado neste trabalho. Os subcritérios foram ponderados em uma escala que varia de 1 a 10 , em que 1 indica alta adequabilidade para o recebimento do empreendimento e 10, baixa adequabilidade. Os valores encontrados foram multiplicados e normalizados para uma escala de 1 a 100 (Tabela 5).

$\mathrm{Na}$ Tabela 5 , observa-se que o critério que obteve a maior ponderação foi a camada de assentamentos rurais (C6), Unidades de conservação ambiental (C1) e Terras indígenas (C3). Segundo a Portaria $n^{\circ} 421$, de 26 de outubro de 2011, estas áreas devem ser evitadas, mostrando que o processo de ponderação apresenta consistência.

Observa-se que os aspectos ambientais receberam as maiores ponderações, seguidos pelos sociais e econômicos. Na prática, segundo os técnicos entrevistados, para fins de definição do traçado de diretrizes de LT os aspectos ambientais e sociais apresentam maior relevância que os econômicos, demonstrando que o processo de ponderação apresenta consistência.

Com a tarefa de ponderação concluída, iniciou-se o processo de reclassificação dos dados para posteriormente aplicação do processo de álgebra de mapas com a utilização do software GRASS 7.6.1. Nesta etapa foram construídas 3 superfícies de custo discreto intermediárias, uma para cada grupo considerado neste trabalho (Figura 4).

$\mathrm{Na}$ Figura 4, observa-se que as áreas em vermelho são consideradas de baixa adequabilidade para o recebimento de uma LT de energia elétrica, enquanto as áreas em azul se apresentam adequadas para receber 0 empreendimento em questão.

A partir do processo de álgebra de mapas, realizou-se a junção das três superfícies em uma única, a fim de se obter a superfície de custo total para a área de influência de projeto da LT (Figura 5).

Baseado na superfície, apresentada na Figura 5 , e as coordenadas do ponto de início e de destino do traçado da diretriz, iniciou-se a construção da superfície de custo acumulado, a qual é organizada em uma estrutura de grafos em que as arestas são ponderadas com a média dos valores de adequabilidade de dois pixels vizinhos e aos vértices são atribuídas as coordenadas do centroide de cada pixel pertencente a matriz de adequabilidade. Este processo foi realizado no software GRASS 7.4.6. 
Tabela 5. Ponderações finais (P1: Pesos atribuído aos grupos, P2: Pesos atribuídos aos critérios e P3: Pesos atribuídos aos subcritérios).

\begin{tabular}{|c|c|c|c|c|c|c|c|}
\hline Grupo & P1 & Critério & $\mathbf{P 2}$ & Subcritério & P3 & $\mathbf{P 1} * \mathbf{P} 2 * \mathbf{P} 3$ & Normalizado \\
\hline \multirow{6}{*}{ Ambiental } & \multirow{6}{*}{34} & \multirow{2}{*}{$\mathrm{C} 1$} & \multirow{2}{*}{35} & $\mathrm{Sim}$ & 10 & 11900 & 84 \\
\hline & & & & Não & 0 & 0 & 0 \\
\hline & & \multirow{2}{*}{$\mathrm{C} 2$} & \multirow{2}{*}{17} & Sim & 6 & 3468 & 25 \\
\hline & & & & Não & 0 & 0 & 0 \\
\hline & & \multirow{2}{*}{$\mathrm{C} 3$} & \multirow{2}{*}{35} & Sim & 10 & 11900 & 84 \\
\hline & & & & Não & 0 & 0 & 0 \\
\hline \multirow{10}{*}{ Social } & \multirow{10}{*}{30} & \multirow{4}{*}{$\mathrm{C} 4$} & \multirow{4}{*}{32} & Área urbana & 10 & 9600 & 68 \\
\hline & & & & Vegetação & 6 & 5760 & 41 \\
\hline & & & & Agricultura & 4 & 3840 & 27 \\
\hline & & & & Água & 10 & 9600 & 68 \\
\hline & & \multirow{4}{*}{$\mathrm{C} 5$} & \multirow{4}{*}{21} & $90 \mathrm{~m}$ & 3 & 5040 & 36 \\
\hline & & & & $180 \mathrm{~m}$ & 2 & 3780 & 27 \\
\hline & & & & $270 \mathrm{~m}$ & 1 & 3150 & 22 \\
\hline & & & & $360 \mathrm{~m}$ & 8 & 1890 & 13 \\
\hline & & \multirow{2}{*}{ C6 } & \multirow{2}{*}{47} & Sim & 10 & 14100 & 100 \\
\hline & & & & Não & 0 & 0 & 0 \\
\hline \multirow{9}{*}{ Econômico } & \multirow{9}{*}{10} & \multirow{5}{*}{$\mathrm{C} 7$} & \multirow{5}{*}{28} & Plano & 1 & 280 & 2 \\
\hline & & & & $\begin{array}{l}\text { Suave } \\
\text { Ondulado }\end{array}$ & 2 & 560 & 4 \\
\hline & & & & Ondulado & 4 & 1120 & 8 \\
\hline & & & & $\begin{array}{l}\text { Forte } \\
\text { Ondulado }\end{array}$ & 5 & 1400 & 10 \\
\hline & & & & Montanhoso & 7 & 1960 & 14 \\
\hline & & \multirow{2}{*}{$\mathrm{C} 8$} & \multirow{2}{*}{19} & Sim & 3 & 570 & 4 \\
\hline & & & & Não & 0 & 0 & 0 \\
\hline & & \multirow{2}{*}{ C9 } & \multirow{2}{*}{53} & Sim & 10 & 5300 & 38 \\
\hline & & & & Não & 0 & 0 & 0 \\
\hline
\end{tabular}
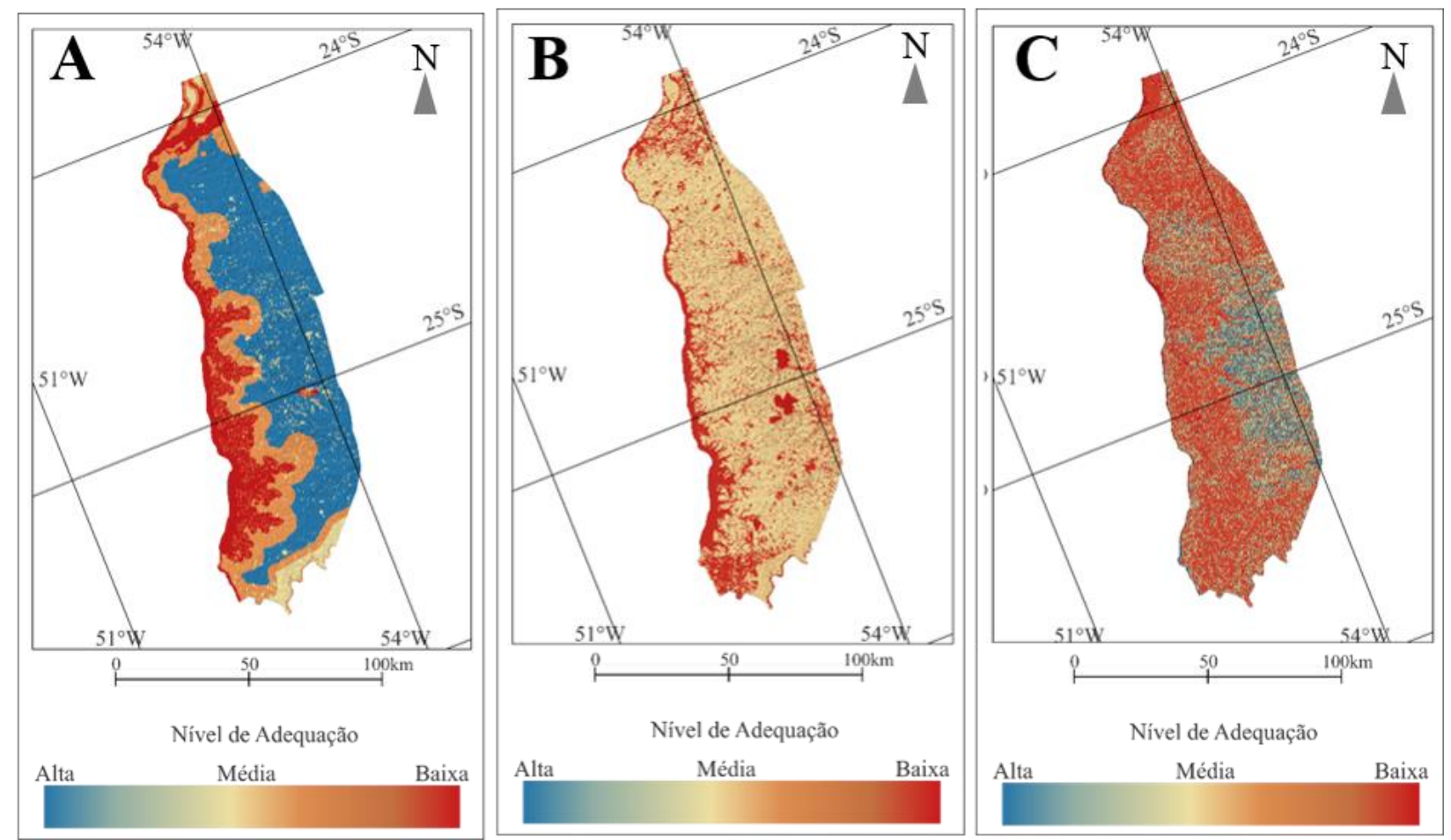

Figura 4. Superfícies intermediárias de custo discreto (A) Ambiental (B) Social (C) Econômico. 
Com a aplicação do algoritmo de Dijkstra (1959), obteve-se o traçado otimizado, ilustrado na Figura 6. O traçado da LT original apresenta

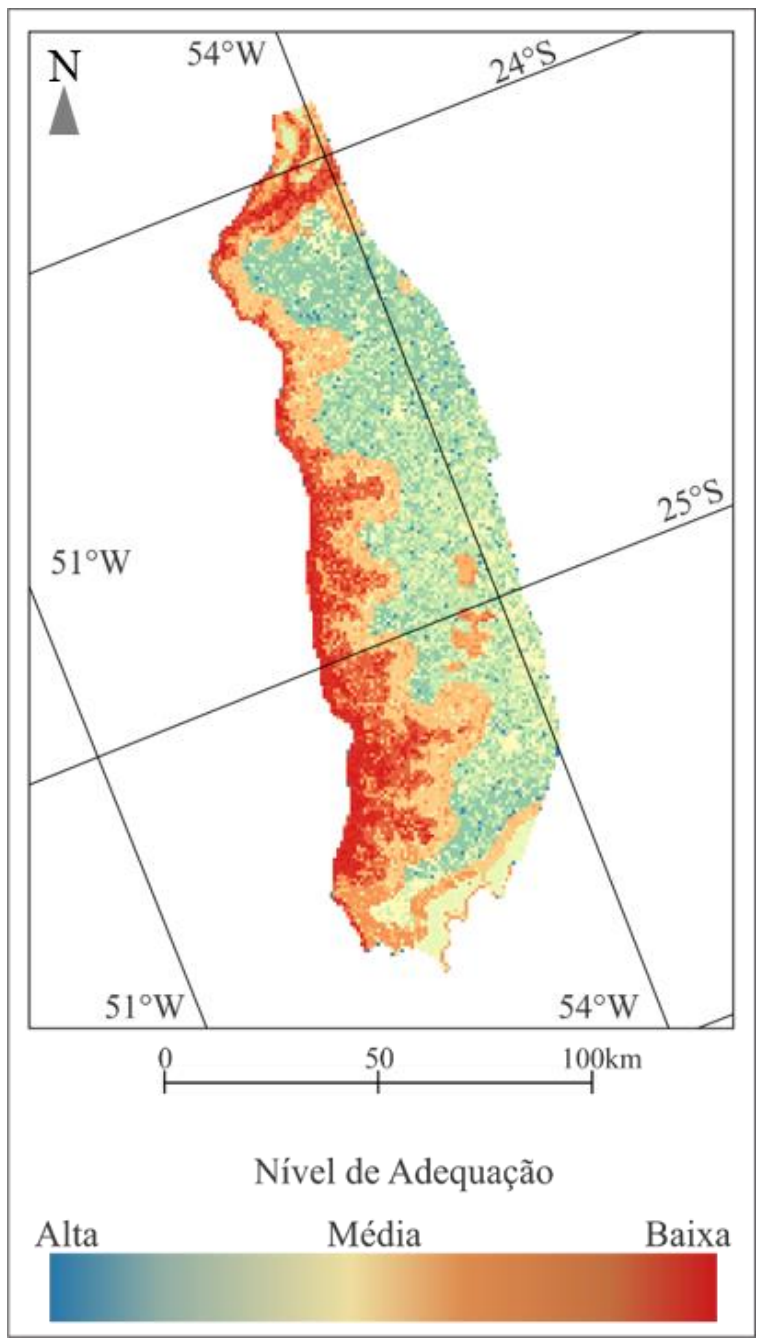

Figura 5. Superfície de custo discreto total.

Observa-se que utilizando a MEM definiu as regiões que apresentaram as características necessárias para o desenvolvimento do projeto, considerando automaticamente uma gama de critérios relevantes no processo de planejamento de diretrizes de LT que, manualmente, seria improvável de serem considerados simultaneamente, possibilitando a realização de análises no entorno do traçado obtido para a identificação de possíveis problemas ou definição de soluções alternativas. Apesar do traçado original e do extensão linear de 172,279 km. Em comparação, o traçado obtido com a aplicação da MEM apresentou extensão linear de 172,603 km.

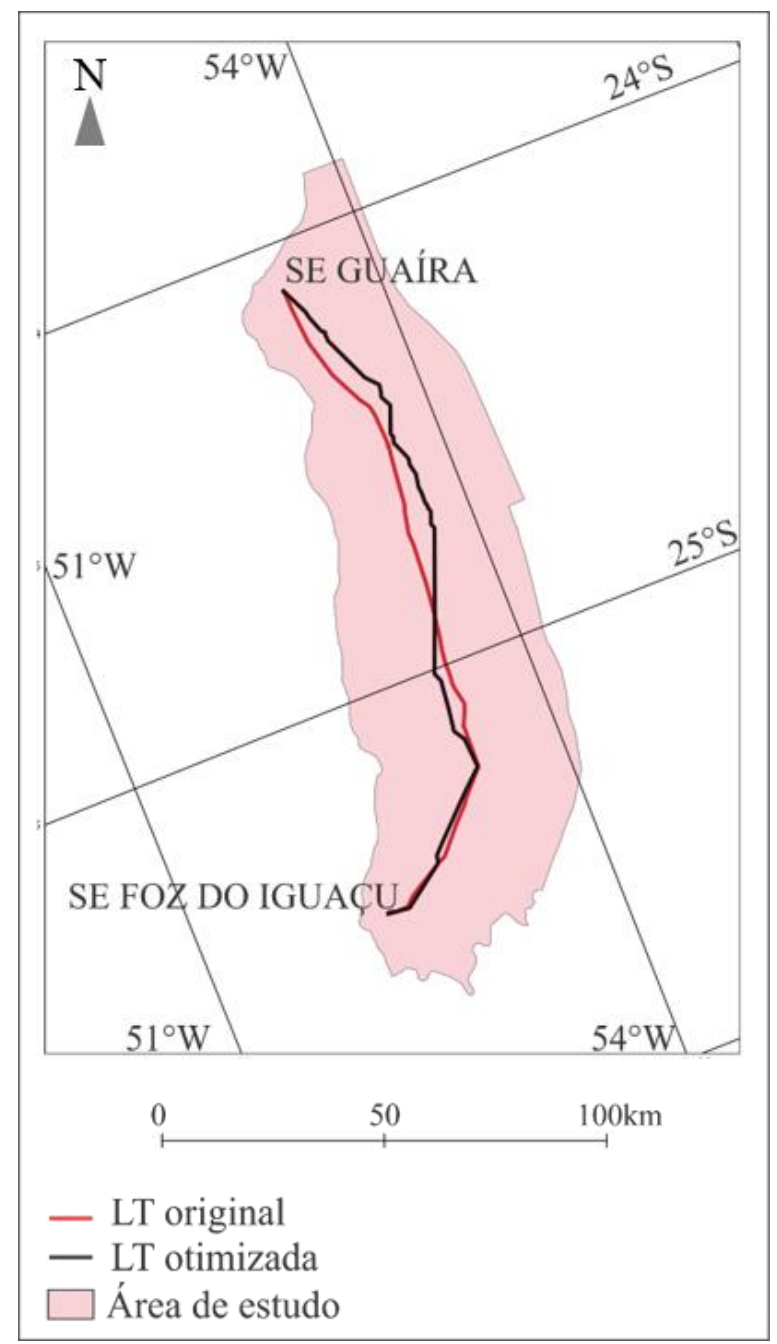

Figura 6. Traçado otimizado.

obtido não serem completamente coincidentes, observa-se que a diferença fica restrita a uma área de $6 \mathrm{~km}$ ao redor do traçado original. Isto permite verificar que a metodologia é passível de aplicação na etapa de planejamento de uma diretriz de LT de energia elétrica, uma vez que a definição de corredores para o planejamento de diretrizes de LT definidos pela ANNEL é de $20 \mathrm{~km}$, permitindo a minimização da área a ser percorrida e o tempo de análise pelos especialistas.

\section{CONCLUSÕES E RECOMENDAÇÕES}

Este trabalho descreveu e testou a combinação entre as ferramentas SIG, dados de Sensoriamento Remoto e o método de análise multicritério hibrido, formado a partir da combinação entre a inferência Fuzzy e o AHP para a definição de diretrizes de LT de energia elétrica.

Os resultados obtidos com a modelagem proposta demonstram que: 1) A aplicação do método Fuzzy AHP permite a definição de diretrizes de LT auxiliando no processo de planejamento locacional de uma LT; 2) O método pode ser aplicado a qualquer localidade, utilizando informações geográficas e softwares SIG gratuitos, sem a necessidade de realização de visitas a campo e aquisição de dados in loco; e 3) concessionárias podem utilizar do processo de 
modelagem espacial multicritério para a definição de áreas adequadas a implantação de uma LT de energia elétrica, minimizando custos de projeto, impactos ambientais e sociais na área de abrangência do empreendimento.

Diante do exposto, observa-se que a técnica empregada para a definição de diretrizes de LT é aplicável na etapa de planejamento locacional de diretrizes de LT, e contribui para a quantificação dos impactos gerados e definição dos traçados potenciais para a realização do processo licitatório do projeto. Além da definição do traçado de uma diretriz preferencial é possível a definição de áreas prioritárias (corredores) para o recebimento de estudos aprofundados acerca de suas características sociais, econômicas e ambientais.

Recomenda-se para trabalhos futuras a realização das ponderações por um número maior de especialistas para a minimização da subjetividade do processo, bem como a inserção de outras informações, como: o valor da terra, propriedades afetadas pelo empreendimento, áreas de alto valor cultural, monetização das variáveis e inserção de dados meteorológicos.

\section{AGRADECIMENTOS}

Os autores externam seus agradecimentos à COPEL Geração e Transmissão S.A. no âmbito do Programa de Pesquisa e Desenvolvimento Tecnológico do Setor de Energia Elétrica, regulamentado pela Agência Nacional de Energia Elétrica - ANEEL pelo auxílio de bolsa do segundo autor no Projeto de P\&D PD-06491-0353-2014.

\section{REFERÊNCIAS}

BACHMANN, D.; BOKLER, F.; KOPEC P.; SCHWARZE B.\& WEICHERT F. 2018. Multi-Objective Optimisation Based Planning of Power-Line Grid Expansions. Isprs International Journal Of Geo-information, 3: 258- 280.

BAGLI, S.; GENELETTI D. \& ORSI F.2011. Routeing of power lines through least-cost path analysis and multicriteria evaluation to minimise environmental impacts. Environmental Impact Assessment Review, 3: 234-239.

DIJKSTRA, E. W,. A note on two problems in connexion with graphs. 1959, Numerische Mathematik, 1(1): 269-271.

DNIT, 2018. Departamento Nacional de Infraestrutura de Transportes. Disponível em: http://www.dnit.gov.br/mapas\%20multimodais/shapefiles. Acesso em: 18 out. 2018.

EPE. 2005. Diretrizes para elaboração dos relatórios técnicos referentes às novas instalações da rede básica. Brasília, 25p.

EPE. 2018. Web Map EPE. Disponível em: https://gisepeprd.epe.gov.br/webmapepe/ Acesso em: 20 out. 2018

GEOINFO. 2019. Infraestrutura de dados espaciais da Embrapa. Disponível em: http://geoinfo.cnpm.embrapa.br/. Acesso em: 8 jun. 2019.

GRASSI, S.; FRIEDLI R.; GRANGIER M. \& RAUBAL M. 2014. A GIS-Based Process for Calculating Visibility Impact from Buildings During Transmission Line Routing. Connecting A Digital Europe Through Location And Place,.1: 383-402.

IAP. 2018. Secretaria do Meio Ambiente e Recursos Hídricos. Disponível em: http://www.iap.pr.gov.br/pagina-756.html Acesso em: 23 out. 2018.

INCRA. 2019. Acervo Fundiário. Disponível em: http://www.acervofundiario.incra.gov.br/ Acesso em: 30 mai. 2019.

LIMA, R. M.; OSIS R.; QUEIROZ A. R. \& SANTOS H. M. 2016. Least-cost path analysis and multi-criteria assessment for routing electricity transmission lines. IET Generation, Transmission \& Distribution, 16: 4222-4230.

MALCZEWSKI，J. 2006 GIS-based multicriteria decision analysis: a survey of the literature. International. Journal Of Geographical Information Science, 7: 703-726.

MINISTÉRIO DO MEIO AMBIENTE (BR). 2011. Portaria $\mathrm{n}^{\circ}$ 421 , de 26 de outubro de 2011. Dispõe sobre o licenciamento e a regularização ambiental federal de sistemas de transmissão de energia elétrica e dá outras providências, Brasil, p. 42.

MIRANDA, E. E. de; (Coord.). 2005. Brasil em Relevo. Campinas: Embrapa Monitoramento por Satélite. Disponível em: https://www.cnpm.embrapa.br/projetos/relevobr/ Acesso em: 30 jan. 2019

MONTEIRO, C.; RAMIREZ-ROSADO I.J.; MIRADNA V.; ZORZANO-SANTAMARIA P.J.; GARCIA-GARRIDO E.; \& FERANDNEZ JIMENEZ L.A. 2005. GIS Spatial Analysis Applied to Electric Line Routing Optimization. IEEE Transactions On Power Delivery, 20 (2):.934-942.

PAPAJOANNOU, G.; VASILIADES, L. \& LOUKAS. 2014. A. Multi-Criteria Analysis Framework for Potential Flood Prone Areas Mapping. Water Resources Management, 29 (2): 399 418.

SAATY, T. L. 1980. The Analytic Hierarchy Process. McGrawHill, New York, NY.

SHU J.; WU L., LI, Z.; SHAHIDEHPOUR M.; ZHANG L. \& HAN B.2012. A New Method for Spatial Power Network Planning in Complicated Environments. IEEE Transactions On Power Systems, 27 (1): 381-389.

TAHA, H. A. 2008. Pesquisa Operacional. São Paulo,: Person Education do Brasil

VERONESI, F., SCHITO, J.; GRASSI S.\& RAUBAL M. 2017. Automatic selection of weights for GIS-based multicriteria decision analysis: site selection of transmission towers as a case study. Applied Geography, 83: 78-85. 